\section{New mouse models of cancer}

\section{By Kai-Jye Lou, Staff Writer}

Researchers at The Salk Institute for Biological Studies have developed an oncogene expression system that generates mouse models that recapitulate tumorigenesis more accurately than xenograft or transgenic approaches. ${ }^{1}$ They have created a mouse model that closely mimics the development and pathophysiology of glioma in humans and are working on a second model for lung cancer.

There are three main types of mouse models of cancer: xenograft models involve the transplantation and growth of human tumor cells in immunodeficient mice; transgenic models involve genetically modifying mice to express oncogenes at the tissue or whole-body level; and genetic knockout models use partial or full deletion of a gene, also at the tissue or whole-body level. None of the models does a good job recapitulating tumorigenesis.

"The current preclinical system we use for drug development just doesn't work-only about $5 \%$ of compounds that make it through preclinical testing make it through clinical trials," said Terry Van Dyke, a professor in the Department of Genetics and Department of Biochemistry and Biophysics at The University of North Carolina at Chapel Hill.

For example, the immune system is knocked out in xenograft models. And because human tumor cells often already carry multiple mutations, it is difficult to pick out the actual cancer-driving mutations using xenograft mice.

Different issues arise with genetically engineered mice. "In most methods, the cancer drivers are expressed in all cells in the mouse," which does not fully represent how cancer arises in humans, said Van Dyke. "Cancer in humans presumably starts in a single cell, starting with a mutation or abnormal condition that gives it a selective advantage over surrounding cells."

To circumvent these issues, a group at the Salk Institute showed in Nature Medicine that lentiviral vector-based oncogene expression in as few as 50 cells can generate cell type-specific cancers in immunocompetent adult mice.

"This method allows you to look much closer at the pathophysiology of the human tumor," said Inder Verma, an American Cancer Society professor at the Salk Institute and principal investigator on the paper. "We know there are many mutations in tumors, but it is not clear which are tumorigenic and which are just background. With these models, we can look at the genetic changes that occur during the progression of a tumor and identify the relevant genes that are turned on."

As proof of concept, the researchers used the lentiviral vector-based system to create a mouse model of glioma that bypassed the deficiencies in xenograft and transgenic mouse models.

In immunocompetent adult mice, injection of lentiviral vectors carrying activated v-Ha-ras Harvey rat sarcoma viral oncogene homolog (HRAS) and protein kinase B (PKB; AKT) into the hippocampus or subventricular zone resulted in tumors similar to human high-grade gliomas. Subsequent injection of as few as 10 glioma cells isolated from the models into the hippocampus of immunodeficient mice led to tumor formation analogous to that triggered by cancer stem cells that initiate brain tumors.

The latter result suggests that every tumor cell isolated from the Salk models has the ability to form a glioma. In contrast, the formation of tumors in xenograft models typically requires at least 10,000 cells.

"The use of lentiviral delivery to a small region in the brain allows for the induction of tumors in adult immunocompetent mice, originating from a few cells, as opposed to transgenic models, in which the tumors often arise from a large number of cells in multiple regions," said David Grass, divisional VP of scientific operations and in vivo discovery at Caliper Life Sciences Inc. Caliper markets a range of xenograft and transgenic mouse models.

Said Van Dyke, "The advantage of including these types of models in studies is that we can see what happens when we express cancer genes in a small number of cells - this is a more accurate recapitulation of how cancer comes about in humans."

Murray Robinson, SVP of oncology at Aveo Pharmaceuticals Inc., said a selling point of Verma's models is their defined genetic context. "Here, they are creating models where they can specifically create alterations in AKT, HRAS and/or p53, and then assess the relevance of each alteration to oncogenesis," he told SciBX. This is in contrast to cells isolated from patient samples and xenograft models, in which the relevant oncogenic alterations are rarely known.

Aveo's lead compound is AV-951, an inhibitor of VEGF receptor 1 (FLT1; EGFR1), VEGF receptor 2 (KDR/FLK-1; VEGFR2) and VEGF receptor 3 (FLT4; VEGFR3). It is in a Phase II trial to treat metastatic renal cell carcinoma (RCC). The company also has developed geneticallydefined, human tissue transgenic mouse models of cancer using a lentiviral vector-based oncogene expression system.

Robinson also liked that the lentiviral vector-based oncogene expression system can generate cancer in adult mice. He said this more closely replicates cancer in humans because oncogenic somatic mutations leading up to the disease typically occur during adulthood.

\section{More human, more cancers}

In addition to making mouse models more human-like, academics contacted by SciBX think the Salk Institute's approach may expand the types of cancer that can be modeled and decrease the effort involved in model generation because the system can be used to transduce oncogenes into both dividing and nondividing mouse cells in vivo.

Avian retroviral vectors typically used to transduce oncogenes in transgenic mouse models are unable to effectively target nondividing cells, such as glial cells in the adult CNS. Moreover, the mouse also 
needs to be genetically modified to express a viral receptor that renders its cells susceptible to infection.

"If you are restricted in the types of cells that you can go into, you may not be able to target all the cells susceptible to cancer in humans," noted Van Dyke.

Eric Holland, director of the Brain Tumor Center and vice chairman for translational research in the Department of Surgery at Memorial Sloan-Kettering Cancer Center, also noted that lentiviral vectors can carry more genetic information than avian retroviral vectors and do not require the extra effort of engineering a viral receptor into transgenic mice.

He cautioned, however, that the native ability of lentivirus to infect mammalian cells carries potential safety concerns. He said the avian retrovirus-transgenic mouse pairing is less likely to cause cross-species infection.

\section{Barriers to adoption}

Other hurdles may remain to adopting widespread use of the Salk Institute's models.

Van Dyke noted that xenograft models are widely used because of their simplicity and reproducibility, whereas models that more accurately approximate a disease-such as the Salk approach-tend to be more complex. "This means that generation of the model would likely require an expert, which would make such models less amenable for widespread use," she said. "The question is: how can you simplify the method without simplifying the disease?"

Robinson also cautioned that disease models developed in the academic setting are "rarely adapted for use in an industry context. People haven't taken the next steps-these primary models need to be adapted for use in drug studies. One could treat one of these tumors with a drug, but the information gained from that one experiment is really limited. Ideally, they would need to grow enough of the tumor to be able to characterize it, freeze it down and have enough of that material to run hundreds of tests. Then it would be a powerful tool for drug discovery and translational research."

Likewise, Holland said he wanted to see whether Verma's lentiviral-based system is reproducible and how reliably oncogene transduction results in actual tumorigenesis. "We want to be able to reliably generate large populations of mice that have the same tumors," he said.

Verma says his group is now using the lentiviral vector-based oncogene expression system to generate a mouse lung cancer model. A patent application covering the mouse models and the lentiviral vector-based oncogene expression methodology has been filed. The work is available for licensing from The Salk Institute Office of Technology Management and Development.

Lou, K.-J. SciBX 2(5); doi:10.1038/scibx.2009.170

Published online Feb. 5, 2009

\section{REFERENCES}

1. Marumoto, T. et al. Nat. Med.; published online Jan. 4, 2009; doi: $10.1038 / \mathrm{nm} .1863$

Contact: Inder M. Verma, The Salk Institute for Biological Studies, La Jolla, Calif.

e-mail:verma@salk.edu

\section{COMPANIES AND INSTITUTIONS MENTIONED}

American Cancer Society, Atlanta, Ga.

Aveo Pharmaceuticals Inc., Cambridge, Mass.

Caliper Life Sciences Inc. (NASDAQ:CALP), Hopkinton, Mass.

Memorial Sloan-Kettering Cancer Center, New York, N.Y.

The Salk Institute for Biological Studies, La Jolla, Calif.

The University of North Carolina at Chapel Hill, Chapel Hill, N.C. 